\title{
Anionic Polymerization of Vinyl Monomers with Organometallic Compound-Phosphoramide Systems
}

\author{
Masao Tomoi, Teruo Onozawa, Kenji Seki, and Hiroshi KaKiuchi \\ Department of Applied Chemistry, Faculty of Engineering, \\ Yokohama National University, Yokohama, Japan.
}

(Received October 28, 1974)

\begin{abstract}
The polymerization of vinyl monomers with organometallic compounds was carried out in the presence of monodentate or bidentate phosphoryl compounds with $-\mathrm{N}\left(\mathrm{CH}_{3}\right)_{2}$ substituents. The polymerization of styrene or $\alpha$-methylstyrene $(\alpha$-MeSt $)$ with organomagnesium compounds was more facilitated in the presence of the bidentate donors than in the presence of the monodentate type. In the polymerization of acrylonitrile (AN) with diethylzinc or organoaluminium compounds, the bidentate phosphoramides were less effective donors than the monodentate ones. The latter polymerization was considered to be caused by the coordination of both the phosphoramides and AN with the organometallic compounds, and to be reduced by the undesirable coordination of the bidentate phosphoramides to the sites required for the coordination of $\mathrm{AN}$. The equilibrium monomer concentration $\left([\mathrm{M}]_{\mathrm{e}}\right)$ in the polymerization of $\alpha$-MeSt was dependent on the solvents used for the polymerization; the values of $[\mathrm{M}]_{\mathrm{e}}$ was smaller in toluene than in tetrahydrofuran.
\end{abstract}

KEY WORDS Anionic Polymerization / Vinyl Monomer / Organometallic Compound / Phosphoramides / Coordination / Initiator Efficiency / Equilibrium Monomer Concentration / Branched Polymer / Molecular Weight Distribution /

Organometallic compounds, which by themselves have no ability to initiate the polymerization of vinyl monomers, were found to cause polymerization in the presence of hexamethylphosphoramide (HMPA), e.g., in the case of organomagnesium compounds-styrene or $\alpha$ methylstyrene, ${ }^{1,2}$ alkali metal alkoxides-methyl methacrylate, ${ }^{3}$ and organoaluminium compounds-acrylonitrile ${ }^{4,5}$ or methyl methacrylate. ${ }^{5}$ Such a change in the reactivity of the organometallic compounds has been considered to be caused by the coordination of HMPA with the metal atoms of the compounds. ${ }^{1-5}$ That is to say, such a coordination results in increases in the polarity of carbon (or oxygen)-metal bonds of the compounds and thereby in the nucleophilicity of the organic groups in the compounds:

$$
\begin{aligned}
& \mathrm{C}^{\delta-}-\mathrm{M}^{\delta+}+m \mathrm{HMPA} \longrightarrow \\
& \mathrm{C}^{\delta-} \beth \mathrm{M}^{\delta+} \longleftarrow \text { mHMPA } \\
& \text { more polar species }
\end{aligned}
$$

The reactivity of the compounds has been also reported to increase in the presence of Lewis bases such as dipyridyl, ${ }^{6}$ triphenylphosphine, ${ }^{6}$ and dimethylsulfoxide ${ }^{3,7}$ or alkoxides such as 2 alkoxyethoxides. ${ }^{8}$

In this paper, the polymerization of some vinyl monomers with organometallic compounds was carried out in the presence of bidentate phosphoryl compounds with $-\mathrm{N}\left(\mathrm{CH}_{3}\right)_{2}$ substituents in order to investigate the effect of phosphoramides on the reactivity of the organometallic compounds.

\section{EXPERIMENTAL}

\section{Reagents}

Nonamethylimidodiphosphoramide (NIPA) and octamethylpyrophosphoramide (OMPA) were<smiles>[X]P(=O)(N(C)C)N(C)C</smiles>

$\mathrm{X}=\mathrm{NCH}_{3}$, NIPA; $\mathrm{X}=\mathrm{O}$, OMPA. 
Polymerization with Organometallic Compounds

obtained via the following synthetic routes: ${ }^{9,10}$

(a) $\mathrm{HMPA} \stackrel{\mathrm{POCl}_{3}}{\longrightarrow}\left[\left(\mathrm{CH}_{3}\right)_{2} \mathrm{~N}\right]_{2} \mathrm{POCl}$

(I)

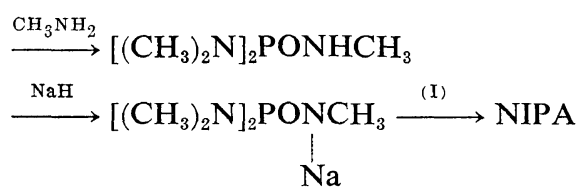

(b) (I) $\stackrel{\mathrm{NaOH}}{\longrightarrow}$ OMPA

Hexamethylthiophosphoramide (HMTP) ${ }^{11}$ was synthesized by the reaction of tris(dimethylamino)phosphine ${ }^{12}$ with sulfur. Dibenzylmagnesium was prepared from the reaction of dibenzylmercury ${ }^{13}$ with magnesium in dry ether under a nitrogen atmosphere at room temperature. ${ }^{14}$ Diethylzinc was prepared by the method described in the literature. ${ }^{15}$ Organoaluminium compounds were obtained as $n$-hexane solutions (15\%) from commercial sources. Acrylonitrile (AN) was freed of inhibitor and traces of water by refluxing and distillation from $\mathrm{ca}$. $5 \%$ by weight of phosphorus pentoxide under a nitrogen atmosphere just before use. ${ }^{16}$ The other reagents were purified by the methods described in previous papers. ${ }^{1,2}$

\section{Polymerization Procedure}

The polymerization of styrene (St) or $\alpha$ methylstyrene $(\alpha$-MeSt) was carried out according to the previously described procedure. ${ }^{1,2}$ In the case of the polymerization of AN, the polymerization mixture was dissolved, after a given time, in $c a .10 \mathrm{ml}$ of dimethylformamide (DMF) containing a small amount of methanol, and poured into methanol. The precipitated polymer was separated by filtration, washed with methanol, and dried under vacuum at $50^{\circ} \mathrm{C}$.

\section{Measurements}

The reduced viscosities of the polymer solutions were measured using DMF as a solvent at $25^{\circ} \mathrm{C}$. Molecular weights and molecular weight distributions of polymers were measured on a Shimadzu GPC-1A gel-permeation chromatograph in tetrahydrofuran (THF) at room temperature with an elution rate of approximately $1 \mathrm{ml} / \mathrm{min}$. Initiator efficiency, $f$, was determined from the yields and the degrees of polymerization of the polymers.

\section{RESULTS AND DISCUSSION}

\section{Polymerization of St or $\alpha$-MeSt with Organomag-} nesium Compound-Phosphoramide Systems

Equilibrium Polymerization of $\alpha$-MeSt. It is well-known that the polymerization of $\alpha$-MeSt with organo-alkali metal compounds is a living polymerization of an equilibrium type. ${ }^{17,18}$ The polymerization with Grignard's reagent-HMPA systems in toluene also showed a similar behavior, but the monomer concentration in equilibrium, $[\mathrm{M}]_{\mathrm{e}}$, was slightly lower than that in the case of the polymerization with organo-alkali metal compounds in THF. ${ }^{2}$ Table I shows the results of the polymerization of $\alpha$-MeSt in both toluene and THF. The value of $[\mathrm{M}]_{\mathrm{e}}$ obtained in toluene was $c a .0 .55 \mathrm{M}$ at $0^{\circ} \mathrm{C}$, while that obtained in THF ca. $0.74 M$. The latter value is in close agreement with that reported in the literature $\left([\mathrm{M}]_{\mathrm{e}}=c a .0 .72 \mathrm{M}\right.$ at $\left.0^{\circ} \mathrm{C}\right) .^{17}$ The polymerization in dioxane also has been reported to give an approximately $20 \%$ lower value of $[\mathrm{M}]_{\mathrm{e}}$ than that in THF: such an effect of the solvents was explained in terms of a solvent-monomer and solvent-polymer interaction parameter. ${ }^{19}$ The difference in $[M]_{e}$ observed in our study is also considered to be due to such interaction parameters of toluene and THF.

Influence of Structure of Phosphoramides. The bidentate phosphoramides, such as NIPA and OMPA, have been reported to be strongly coordinating ligands for many metal ions, and to yield octahedrally solvated cations in many cases. $^{20-22}$ The coordination of the ligand takes

Table I. Equilibrium polymerization of $\alpha-\mathrm{MeSt}^{\mathrm{a}}$

\begin{tabular}{llccc}
\hline Solvent & Donor & $\begin{array}{c}{[\text { Donor }} \\
{[\text { Initiator }]}\end{array}$ & $\begin{array}{c}\text { Yield, } \\
\%\end{array}$ & $\begin{array}{c}{[\mathrm{M}]_{\mathrm{e}}, \mathbf{b}} \\
M\end{array}$ \\
\hline Toluene & HMPA & 2.0 & 73.7 & 0.57 \\
Toluene & HMPA & 4.0 & 75.4 & 0.54 \\
THF & HMPA & 2.0 & 66.2 & 0.74 \\
THF & HMPA & 4.0 & 66.3 & $0.73_{5}$ \\
Toluene & OMPA & 0.5 & 76.2 & 0.52 \\
Toluene & OMPA & 2.0 & 73.7 & 0.57 \\
THF & OMPA & 0.5 & 64.7 & 0.77 \\
THF & OMPA & 1.0 & 66.0 & 0.74 \\
\hline
\end{tabular}

a Polymerization conditions: $\left[\mathrm{M}_{0}\right], 2.18 M ;\left[\left(\mathrm{C}_{6} \mathrm{H}_{5}\right.\right.$ $\left.\mathrm{CH}_{2}\right)_{2} \mathrm{Mg}$ ], $1.02 \times 10^{-2} \mathrm{M}$; temp, $0^{\circ} \mathrm{C}$; time, $18 \mathrm{hr}$.

b Equilibrium monomer concentration. 
place via the oxygen atoms of phosphoryl groups of the ligand as well as in the case of HMPA. ${ }^{23}$ The X-ray structure analysis of the tris-OMPA complex of magnesium perchlorate showed the presence of three planar chelate rings in the complex. ${ }^{22}$ Such a chelate ring was also found to exist in the complexes of many metal ions with NIPA by measuring their IR and ligandfield spectra. ${ }^{21}$ Therefore, the interaction of one molecule of NIPA or OMPA with organometallic compounds must be stronger than that of two HMPA molecules with the compounds since a six-membered chelate ring can be formed in the former case.

Figure 1 shows the effect of the structure of phosphoramide donors on the polymerization of St with benzylmagnesium bromide. Since the ratio of phosphoryl group to magnesium atom, $[\mathrm{P}=\mathrm{O}] /[\mathrm{Mg}]$, is an important factor for the polymerization, ${ }^{1,2}$ the polymerization behavior at a constant $[\mathrm{P}=\mathrm{O}] /[\mathrm{Mg}]$ ratio was examined. The polymerization proceeded very rapidly in the presence of OMPA at $0^{\circ} \mathrm{C}$, but rather slowly in the presence of HMPA. This fact indicates that OMPA is much more effective in promoting the polymerization than is HMPA.

Table II shows the effect of the $[\mathrm{P}=\mathrm{O}] /[\mathrm{Mg}]$ ratio on the polymerization of St. The polymerization in the presence of HMPA proceeded only at $[\mathrm{P}=\mathrm{O}] /[\mathrm{Mg}]>2$, but in the presence of
OMPA proceeded appreciably even at $[\mathrm{P}=\mathrm{O}] /$ $[\mathrm{Mg}]=1.5$. An increase in the $[\mathrm{P}=\mathrm{O}] /[\mathrm{Mg}]$ ratio resulted in an increase in initiator efficiency, especially in the presence of OMPA. These results also suggest that OMPA is a more efficient donor than HMPA. The large rate of polymerization in the presence of OMPA (Figure 1) is considered to be, at least in part, due to an increase in $f$, i.e., an increase in the concentration of active species formed from the initiator system.

The ratio of the rate constant of propagation

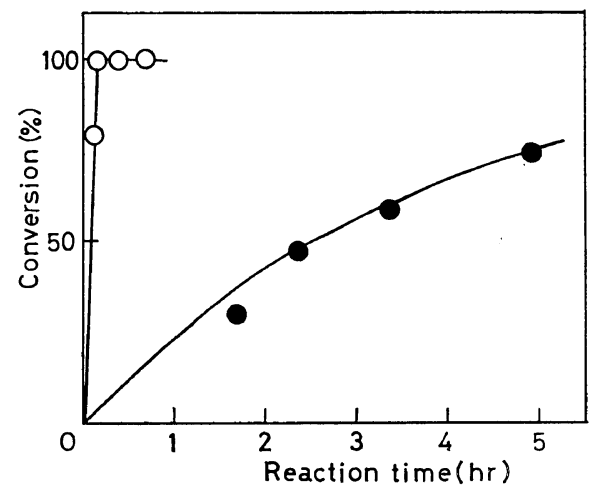

Figure 1. Effect of donors on the polymerization of $\mathrm{St}$ with benzylmagnesium bromide: $[\mathrm{M}], 1.41 \mathrm{M}$; $\left[\mathrm{C}_{6} \mathrm{H}_{5} \mathrm{CH}_{2} \mathrm{MgBr}\right], \quad 7.77 \times 10^{-3} \mathrm{M} ; \quad[\mathrm{P}=\mathrm{O}] /[\mathrm{Mg}]=4.0$; (๑), HMPA; (O), OMPA; solvent, toluene; temp, $0^{\circ} \mathrm{C}$.

Table II. Polymerization of St by benzylmagnesium bromide with phosphoramide donors ${ }^{2}$

\begin{tabular}{|c|c|c|c|c|c|c|c|}
\hline Donor & $\frac{[\mathrm{P}=\mathrm{O}]^{\mathrm{b}}}{[\mathrm{Mg}]}$ & $\begin{array}{c}\text { Yield, } \\
\%\end{array}$ & $\begin{array}{c}M_{n} \\
\times 10^{-4}\end{array}$ & $\begin{array}{c}M_{w} \\
\times 10^{-4}\end{array}$ & $\frac{M_{w}}{M_{n}}$ & $f^{c}$ & $\frac{k_{\mathrm{p}}^{\mathrm{d}}}{k_{\mathrm{i}}}$ \\
\hline HMPA & 2.0 & 0 & - & - & - & - & - \\
\hline HMPA & 2.5 & 10.6 & 1.94 & 3.94 & 2.03 & 0.05 & 7400 \\
\hline HMPA & 3.0 & 75.9 & 6.79 & $14.4_{5}$ & 2.13 & $0.10_{5}$ & 11700 \\
\hline HMPA & 4.0 & 94.2 & 7.42 & 13.2 & 1.78 & 0.12 & 11000 \\
\hline HMPA & 5.0 & 99.8 & 7.06 & 10.7 & 1.51 & 0.13 & 9700 \\
\hline OMPA & 1.0 & 0 & - & - & - & - & - \\
\hline OMPA & 1.5 & 26.7 & 4.66 & 12.7 & 2.72 & 0.05 & 18600 \\
\hline OMPA & 2.0 & 100 & 3.32 & 5.27 & 1.58 & 0.28 & 1860 \\
\hline OMPA & 3.0 & 100 & 2.09 & 3.23 & $1.54_{5}$ & 0.45 & 610 \\
\hline OMPA & 4.0 & 100 & $1.56_{5}$ & 2.73 & $1.74_{5}$ & 0.60 & 285 \\
\hline OMPA & 5.0 & 100 & 1.50 & 2.70 & 1.80 & 0.63 & 247 \\
\hline
\end{tabular}

a Polymerization conditions: $[\mathrm{M}], 1.41 \mathrm{M}$; $\left[\mathrm{C}_{6} \mathrm{H}_{5} \mathrm{CH}_{2} \mathrm{MgBr}\right], 1.56 \times 10^{-2} \mathrm{M}$; solvent, toluene; temp, $0^{\circ} \mathrm{C}$; time, $19 \mathrm{hr}$.

b Ratio of phosphoryl group to initiator.

c Initiator efficiency.

d Ratio of propagation to initiation rate constant. 
$\left(k_{\mathrm{p}}\right)$ to that of initiation $\left(k_{\mathrm{i}}\right)$ was determined by use of the equation derived by Levy and Szwarc. $^{24}$ The values of the $k_{\mathrm{p}} / k_{\mathrm{i}}$ ratio show that the initiation step of the polymerization in the presence of HMPA was much slower than the propagation, and in the presence of OMPA the initiation was facilitated more significantly than the propagation with increasing donor concentration. The molecular weight distributions of the polymers obtained were rather broad compared with those of the polymers obtained by a typical living polymerization. ${ }^{25}$ It is wellknown that in a living polymerization the molecular weight distribution becomes narrow with a decrease in the $k_{\mathrm{p}} / k_{\mathrm{i}}$ ratio. $^{26}$ Such a relation, however, was not observed in this case: a decrease in the $k_{\mathrm{p}} / k_{\mathrm{i}}$ ratio did not always result in a decrease in the value of $M_{w} / M_{n}$, the ratio of the weight-average to number-average molecular weight, which is commonly used as a measure of the polydispersity of a polymer. Other factors, such as the presence of traces of impurities or the inefficient mixing of reagents, might lead to a broadening of the distribution, ${ }^{27}$ because the polymerization was initiated by introducing an initiator solution with a syringe.
Table III shows the results of the polymerization of St with dibenzylmagnesium. This initiator could initiate the polymerization in the presence of HMPA even at $[\mathrm{P}=\mathrm{O}] /[\mathrm{Mg}]<2$ in contrast to the benzylmagnesium halides, which could not initiate the polymerization under similar conditions. ${ }^{1}$ This fact suggests that dibenzylmagnesium is more easily activated by coordination with HMPA than are the benzylmagnesium halides. That is to say, the former could be changed by coordination with one or two HMPA molecules to an active species with ability to initiate the polymerization of St, while the latter could only be changed to such an active species by coordination with four HMPA molecules. $^{2}$

The initiator efficiency increased with increasing $[\mathrm{P}=\mathrm{O}] /[\mathrm{Mg}]$ ratio, especially in the $[\mathrm{P}=\mathrm{O}] /$ $[\mathrm{Mg}]$ range of $0-c a .2$. The bidentate donors were more effective additives also in this polymerization system. The value of the $k_{\mathrm{p}} / k_{\mathrm{i}}$ ratio was significantly larger than that in the case of the polymerization with benzylmagnesium bromide (see Table II). This finding also indicates that the reactivity behavior of dialkylmagnesium compounds is not always in agreement with that

Table III. Polymerization of St by dibenzylmagnesium with phosphoramide donors ${ }^{a}$

\begin{tabular}{lccccccc}
\hline Donor & $\begin{array}{c}{[\mathrm{P}=\mathrm{O}]^{\mathrm{b}}} \\
{[\mathrm{Mg}]}\end{array}$ & $\begin{array}{c}\text { Yield, } \\
\%\end{array}$ & $\begin{array}{c}M_{n} \\
\times 10^{-4}\end{array}$ & $\begin{array}{c}M_{w} \\
\times 10^{-4}\end{array}$ & $\frac{M_{w}}{M_{n}}$ & $f^{\mathrm{c}}$ & $\begin{array}{c}\frac{k}{\mathrm{p}}^{\mathrm{d}} \\
k_{\mathrm{i}}\end{array}$ \\
\hline HMPA & 0.5 & 94.2 & 27.1 & 45.4 & 1.68 & 0.04 & 54500 \\
HMPA & 1.0 & 100 & 16.9 & 24.3 & 1.44 & $0.06_{5}$ & 47300 \\
HMPA & 2.0 & 100 & 13.5 & 20.8 & 1.54 & 0.08 & 30600 \\
HMPA & 4.0 & 99.2 & 12.2 & 18.9 & 1.55 & 0.09 & 25200 \\
HMPA & 10.9 & 99.1 & 13.15 & 22.5 & 1.71 & 0.08 & 28700 \\
OMPA & 0.5 & 98.9 & 11.6 & 19.7 & 1.69 & 0.09 & 22900 \\
OMPA & 1.0 & 100 & 8.22 & 13.4 & 1.63 & 0.13 & 10800 \\
OMPA & 2.0 & 100 & 7.00 & 12.75 & 1.82 & $0.15_{5}$ & 7800 \\
OMPA & 6.0 & 100 & 5.65 & 9.38 & 1.66 & 0.19 & 4900 \\
OMPA & 10.0 & 100 & 5.05 & 8.34 & 1.65 & $0.21_{5}$ & 3850 \\
NIPA & 0.5 & 97.7 & 13.6 & 29.9 & 2.21 & 0.08 & 32000 \\
NIPA & 1.0 & 94.2 & 6.84 & 20.4 & 2.98 & 0.15 & 7900 \\
NIPA & 2.0 & 95.5 & 5.47 & 12.9 & 2.35 & 0.19 & 4850 \\
NIPA & 6.0 & 98.9 & 4.16 & 9.17 & 2.20 & 0.26 & 2550 \\
NIPA & 10.0 & 98.5 & 3.52 & 7.92 & 2.25 & 0.31 & 1730 \\
\hline
\end{tabular}

a Polymerization conditions: $[\mathrm{M}], 1.41 \mathrm{M} ;\left[\left(\mathrm{C}_{6} \mathrm{H}_{5} \mathrm{CH}_{2}\right)_{2} \mathrm{Mg}\right], 1.35 \times 10^{-2} M$; solvent, toluene; temp, $0^{\circ} \mathrm{C}$; time, $19 \mathrm{hr}$.

b Ratio of phosphoryl group to magnesium of initiator.

- Initiator efficiency estimated by assuming that dibenzylmagnesium acts as a monofunctional initiator.

d Ratio of propagation to initiation rate constant. 
of the corresponding Grignard's reagents. A similar disagreement is also observed in the polymerization of methyl methacrylate with organo-magnesium compounds: Grignard's reagents derived from alkyl bromides exerted a more pronounced stereoregulating effect than did the dialkylmagnesium compounds. ${ }^{28}$

An increase in the $k_{\mathrm{p}} / k_{\mathrm{i}}$ ratio had little or no effect on the molecular weight distribution of the polymer. The polymer formed in the presence of NIPA, however, had a broader distribution than that obtained in the presence of HMPA or OMPA. In the presence of NIPA, both benzyl groups of a part of the initiator may take part in the initiation with distinct rates because of the stronger coordinating power of the donor, and hence the distribution may broaden. The slightly lower yields of the polymers in the presence of NIPA are considered to come from the cut-off of lower molecular weight polymers at the stage of polymer separation.

The polymerization of St with dibenzylmagnesium was also carried out in the presence of HMTP (Table IV). The polymerization occurred only in the presence of fairly large amounts of HMTP, in contrast to the polymerization in the presence of HMPA. This fact must be due to the weak interaction of HMTP with the organomagnesium compound. Bases in which a donor atom is $\mathbf{S}$ or O have been classified as "soft" or "hard" bases, respectively, by Pearson. ${ }^{29}$ HMTP has been reported to behave as a soft base and to form stable coordination compounds with soft metal ions such as $\mathrm{Hg}(\mathrm{II}), \operatorname{Ag}(\mathrm{I})$, and $\operatorname{Pd}(\mathrm{II})$ via the sulfur atom of the ligand. ${ }^{30}$ On the other hand, magnesium ion has been classified as a hard acid. The magnesium atoms of organometallic compounds also could be classified as hard acids, though the magnesium-carbon bond of the compounds is not a perfectly ionic bond but a polarized one. ${ }^{31}$ Therefore, it is concluded on the basis of "The Principle of Hard and Soft Acids and Bases" (HSAB) ${ }^{29}$ that organomagnesium compounds interact with HMPA more strongly than with HMTP. Actually, the chemical shift of methylene protons for diethylmagnesium moved upfield only slightly in the presence of HMTP, i.e., $\Delta \delta=-0.03$ at $[\mathrm{HMTP}] /[\mathrm{Mg}]=6$, but moved noticeably in the presence of HMPA, i.e., $\Delta \delta=-0.23$ at [HMPA] $[\mathrm{Mg}]=\mathrm{ca} .6 .^{32}$

Figure 2 shows the effect of the donors on the polymerization of $\alpha$-MeSt with dibenzylmagnesium. The value of $f$ increased with an increase in the $[\mathrm{P}=\mathrm{O}] /[\mathrm{Mg}]$ ratio. The value changed markedly in the range of $[\mathrm{P}=0] /[\mathrm{Mg}] 1-2$, when the polymerization was carried out in the presence of the bidentate donors at $0^{\circ} \mathrm{C}$. This finding suggests that the complex of dibenzylmagnesium with one molecule of NIPA or OMPA can initiate the polymerization of $\alpha$-MeSt. The value of $f$ obtained in the presence of the bidentate donor became greater than unity at higher $[\mathrm{P}=\mathrm{O}] /[\mathrm{Mg}]$ ratios. This fact shows that dibenzylmagnesium may act as a bifunctional initiator under these conditions. The large value

Table IV. Polymerization of St with dibenzylmagnesium in the presence of HMTPa

\begin{tabular}{|c|c|c|c|c|c|c|c|c|}
\hline Solvent & $\frac{[\mathrm{HMTP}]^{\mathrm{b}}}{[\mathrm{Mg}]}$ & $\underset{{ }^{\circ} \mathrm{C}}{\text { Temp }}$ & $\underset{\%}{\text { Yield, }}$ & $\begin{array}{c}M_{n} \\
\times 10^{-5}\end{array}$ & $\begin{array}{c}M_{w} \\
\times 10^{-5}\end{array}$ & $\frac{M_{w}}{M_{n}}$ & $f^{\mathrm{c}}$ & $\begin{array}{c}{\frac{k_{\mathrm{p}}}{k_{\mathrm{i}}}}_{\times 10^{-4}}\end{array}$ \\
\hline Toluene & 52.7 & 0 & 0 & - & - & - & - & - \\
\hline Toluene & 52.7 & Room temp & 0 & - & - & - & - & - \\
\hline Toluene & 105 & 0 & 93.5 & 4.21 & 8.33 & 1.98 & 0.04 & 19.7 \\
\hline Toluene & 105 & Room temp & 96.0 & 2.62 & 6.42 & 2.45 & $0.06_{5}$ & 7.36 \\
\hline THF & 26.4 & 0 & 0 & - & - & - & - & - \\
\hline THF & 52.7 & 0 & 43.0 & 1.24 & 3.15 & 2.54 & 0.06 & 3.82 \\
\hline THF & 105 & 0 & 95.7 & 2.09 & 3.56 & 1.70 & 0.08 & 4.75 \\
\hline
\end{tabular}

a Polymerization conditions: [M], 2.43M; $\left[\left(\mathrm{C}_{6} \mathrm{H}_{5} \mathrm{CH}_{2}\right)_{2} \mathrm{Mg}\right], 1.44 \times 10^{-2} \mathrm{M}$; time, $18 \mathrm{hr}$.

b Molar ratio of HMTP to initiator.

c Initiator efficiency estimated by assuming that dibenzylmagnesium acts as a monofunctional initiator.

d Ratio of propagation to initiation rate constant. 


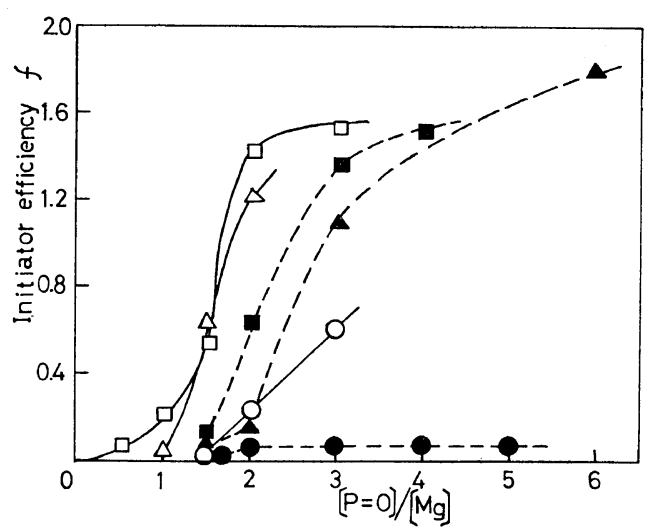

Figure 2. Effect of $[\mathrm{P}=\mathrm{O}] /[\mathrm{Mg}]$ ratio on initiator efficiency in the polymerization of $\alpha$-MeSt with dibenzylmagnesium: [M], 2.12M; $\left[\left(\mathrm{C}_{6} \mathrm{H}_{5} \mathrm{CH}_{2}\right)_{2} \mathrm{Mg}\right.$ ], $1.89 \times 10^{-2} M ;(\bigcirc), \quad$ HMPA, $0^{\circ} \mathrm{C} ;(\bigcirc)$, HMPA, $-20^{\circ} \mathrm{C}$; $(\triangle)$, OMPA, $0^{\circ} \mathrm{C}$; $(\Delta)$, OMPA, $-20^{\circ} \mathrm{C}$; $(\square)$, NIPA, $0^{\circ} \mathrm{C}$; (ロ), NIPA, $-20^{\circ} \mathrm{C}$; solvent, toluene; time $18 \mathrm{hr}$.

of $f$ in this case compared with that in the polymerization of St (see Table III) must be due to the fact that $\alpha$-MeSt has a smaller value of $k_{\mathrm{p}}$ than $\mathrm{St}$ in the case of an anionic polymerization. $^{33}$ The value of $f$ decreased with decreasing polymerization temperature. The activation energy of initiation, therefore, is considered to be larger than that of propagation.

Synthesis of Highly Branched Polystyrene. The synthesis of highly branched polystyrene was tried using vinylbenzylmagnesium chloride as an initiator. The attack of a propagating polystyryl end on the residual vinyl group of the initiator bonded to another polymer was expected to give branched polystyrene. Figure 3 shows the gelpermeation chromatogram of the polymer ob- tained in the presence of HMPA. This polystyrene exhibited an extremely broad molecular weight distribution: the value of $M_{w} / M_{n}$ was ca. 12, while its value was $1.5-2$ in the case of the polymer formed by using benzylmagnesium halides under similar conditions (see Table II). Kurata, et al., have reported that branched polystyrenes prepared by copolymerization of St and 1,4-divinyl-2,3,5,6-tetrachlorobenzene have broad molecular weight distributions; the value of $M_{w} / M_{n}$ increased with an increase in the extent of branching reaction. ${ }^{34}$ The branched polymer, therefore, must have been formed in the polymerization system St-vinylbenzylmagnesium chloride.

\section{Polymerization of AN with Organozinc or Organo- aluminum Compounds}

Table $\mathrm{V}$ shows the results of the polymerization of AN with diethylzinc in the presence of phosphoramides. The polymerization did not occur with diethylzinc alone in toluene at $0^{\circ} \mathrm{C}$. The addition of small amounts of the phosphoramides did not promote the polymerization so much, in contrast to the polymerization with organomagnesium compounds. The yield of poly(AN) increased with an increase in the concentration of the phosphoramides. The bidentate donors, however, were less effective than the monodentate donor, HMPA. This behavior also is in disagreement with that observed in the polymerization of St or $\alpha$-MeSt with organomagnesium compound-phosphoramide systems.

When diethylzinc and NIPA were mixed in toluene, a toluene-insoluble product was obtained. The precipitate, which was separated by filtration, washed with toluene, and dried under vacuum, was found by complexometric titration ${ }^{35}$

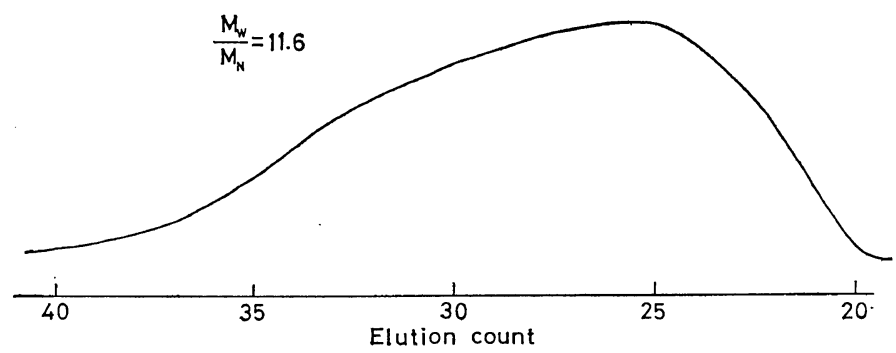

Figure 3. GPC of branched polystyrene: polymerization conditions; [St], 2.46M; $\left[\mathrm{CH}_{2}=\mathrm{CHC}_{6} \mathrm{H}_{4} \mathrm{CH}_{2} \mathrm{MgCl}\right](m, p$-mixture $), 1.63 \times 10^{-2} M$; HMPA, $6.52 \times 10^{-2} M$; solvent, toluene; temp, $0^{\circ} \mathrm{C}$; time $16 \mathrm{hr}$. 
Table V. Polymerization of AN by diethylzinc with phosphoramide donors ${ }^{a}$

\begin{tabular}{lccc}
\hline Donor & $\begin{array}{lccc}{[\mathrm{P}=\mathrm{O}]^{\mathrm{b}}} \\
{[\mathrm{Zn}]}\end{array}$ & $\begin{array}{c}\text { Yield, } \\
\%\end{array}$ & $\begin{array}{c}{\frac{\eta_{\mathrm{sp}}}{c}}_{c}^{\mathrm{c}}, \\
\mathrm{d} l / \mathrm{g}\end{array}$ \\
\hline HMPA & 1.0 & 0 & - \\
HMPA & 2.0 & trace & - \\
HMPA & 5.0 & 15.5 & 0.98 \\
HMPA & 10 & 52.5 & 1.30 \\
HMPA & 20 & 77.7 & 1.23 \\
HMPA & 50 & 91.4 & 0.97 \\
NIPA & 1.0 & 5.0 & 0.25 \\
NIPA & 2.0 & 8.5 & 0.38 \\
& & $(25.8)^{\mathrm{d}}$ & $(0.46)^{\mathrm{d}}$ \\
NIPA & 4.0 & 21.3 & 0.61 \\
NIPA & 10 & 40.6 & 0.90 \\
NIPA & 27 & 61.8 & 1.34 \\
OMPA & 4.0 & 0 & - \\
OMPA & 10 & trace & - \\
OMPA & 20 & 10.4 & 0.43 \\
OMPA & 60 & 28.3 & 0.59
\end{tabular}

a Polymerization conditions: [M], 4.22M; [ $\mathrm{Zn}$ $\left.\left(\mathrm{C}_{2} \mathrm{H}_{5}\right)_{2}\right], 2.55 \times 10^{-2} M$; solvent, toluene; temp, $0^{\circ} \mathrm{C}$; time, $19 \mathrm{hr}$.

b Ratio of phosphoryl group to initiator.

c DMF solution at $25^{\circ} \mathrm{C}, c=0.25 \mathrm{~g} / \mathrm{d} l$.

d Polymerization with $\mathrm{Zn}\left(\mathrm{C}_{2} \mathrm{H}_{5}\right)_{2}$ NIPA complex: [M], 3.95M; [Complex], $8.88 \times 10^{-2} M$; solvent, toluene; temp, room temperature; time, $14 \mathrm{hr}$.

to be the complex with the formula $\mathrm{Zn}\left(\mathrm{C}_{2} \mathrm{H}_{5}\right)_{2}$. NIPA. This complex alone showed a catalytic activity for the polymerization of AN. This fact indicates that the complex must be at least an active species which has the ability to initiate the polymerization.

Table VI shows the results of the polymerization of AN with organoaluminium compounds. The bidentate donors were less effective in promoting the polymerization than HMPA in this case as well as in the case of the polymerization with diethylzinc-phosphoramide systems. In the presence of NIPA, the yield of the polymer increased with increasing NIPA concentration in the range of $[\mathrm{P}=\mathrm{O}] /[\mathrm{Al}] \leq 2$, but a further increase in the concentration, on the contrary, reduced the polymerization. Such a reduction did not take place in the presence of HMPA.

It was confirmed by a copolymerization with St that the polymerization of AN with diethylzinc or organoaluminium compounds-phosphoramide
Table VI. Polymerization of AN by organoaluminium compound with phosphoramide donors

\begin{tabular}{|c|c|c|c|}
\hline Donor & $\frac{[\mathrm{P}=\mathrm{O}]^{\mathrm{b}}}{[\mathrm{Al}]}$ & $\underset{\%}{\text { Yield, }}$ & ${\frac{\eta_{\mathrm{sp}}}{c}}^{\mathrm{c} l / \mathrm{g}}$ \\
\hline \multicolumn{4}{|c|}{ (A) Polymerization with triethylaluminium ${ }^{a}$} \\
\hline HMPA & 0.5 & trace & \\
\hline HMPA & 1.0 & 52.0 & 4.99 \\
\hline HMPA & 2.0 & 63.0 & 5.38 \\
\hline HMPA & 5.0 & 70.2 & 5.28 \\
\hline NIPA & 1.0 & 9.4 & 0.75 \\
\hline NIPA & 2.0 & 19.7 & 1.97 \\
\hline NIPA & 4.0 & 8.5 & 0.73 \\
\hline NIPA & 6.0 & trace & - \\
\hline OMPA & 1.0 & trace & - \\
\hline OMPA & 2.0 & 12.2 & 2.48 \\
\hline OMPA & 4.0 & 15.4 & 2.45 \\
\hline OMPA & 10 & 21.7 & 2.41 \\
\hline
\end{tabular}

(C) Polymerization with triisobutylaluminium ${ }^{\mathrm{d}}$

\begin{tabular}{|c|c|c|c|}
\hline HMPA $^{-}$ & 0.5 & 8.7 & 2.12 \\
\hline HMPA $^{*}$ & 1.0 & 78.4 & 3.48 \\
\hline $\mathrm{HMPA}^{\circ}$ & 2.0 & 79.2 & 3.18 \\
\hline $\mathrm{HMPA}^{\circ}$ & 5.0 & 79.0 & 3.04 \\
\hline NIPA $^{f}$ & 1.0 & 66.0 & 5.35 \\
\hline NIPA $^{f}$ & 2.0 & 80.0 & 2.92 \\
\hline NIPA $^{f}$ & 4.0 & 65.1 & 2.52 \\
\hline NIPA $^{p}$ & 6.0 & trace & - \\
\hline OMPA $^{f}$ & 2.0 & trace & 一 \\
\hline $\mathrm{OMPA}^{\mathrm{f}}$ & 4.0 & 13.3 & 1.78 \\
\hline OMPA $^{f}$ & 6.0 & 22.4 & 2.65 \\
\hline
\end{tabular}

a Polymerization conditions: [M], 4.05M; [Al $\left.\left(\mathrm{C}_{2} \mathrm{H}_{5}\right)_{3}\right]=8.76 \times 10^{-2} M ;$ solvent, toluene; temp, room temperature; time, $19 \mathrm{hr}$.

b Ratio of phosphoryl group to initiator.

c DMF solution at $25^{\circ} \mathrm{C}, c=0.25 \mathrm{~g} / \mathrm{d} l$.

d Polymerization conditions: [M], 3.80M; [Al $\left.\left(i-\mathrm{C}_{4} \mathrm{H}_{9}\right)_{3}\right]=9.46 \times 10^{-2} M$; solvent, toluene; temp, room temperature.

- Reaction time, $2 \mathrm{hr}$.

f Reaction time, $19 \mathrm{hr}$.

systems has essentially an anionic character: the copolymer formed in the equimolar-feed copolymerization had a copolymer composition ( $c a$. 95-\% AN and ca. 5-\% St) similar to that of the copolymer obtained by typical anionic copolymerizations. ${ }^{36}$ This conclusion is in disagreement with the radical mechanism proposed by Akimoto and Okada. ${ }^{5}$ It was observed in our study that these initiator systems could not polymerize methyl methacrylate or St under 
conditions similar to those for the polymerization of AN. This result also cannot be explained in terms of the radical mechanism.

\section{A Consideration Concerning the Interaction of Organometallic Compounds with Phosphoramide \\ Donors}

The reactivities of organometallic compoundphosphoramide systems as initiators for the polymerization of vinyl monomers could be classified into the following two categories: (1) the reactivity increases with an increase in the coordinating power of phosphoramides; (2) the reactivity, on the contrary, decreases with increasing coordinating power of the donors. The polymerization of St or $\alpha$-MeSt with organomagnesium compound-phosphoramide systems belongs to the former class, while the polymerization of AN with organoaluminium compound or diethylzinc-phosphoramide systems is in the latter class.

Such a difference must be due to a difference in structure between the organometallic compounds, especially in the polarity of carbonmetal bonds of the compounds. The electronegativity of magnesium in diethylmagnesium was estimated to be 0.94 from NMR data, ${ }^{32}$ and Pauling's value for the magnesium atom is $1.2{ }^{37}$ The magnesium atom in the organometallic compounds, therefore, is considered to behave as a more hard acid, and, on the basis of the principle of $\mathrm{HSAB},{ }^{29}$ to interact strongly with the phosphoramides. Actually the electronegativity of magnesium in diethylmagnesium decreased in the presence of HMPA (ca. 0.79 at $[\mathrm{HMPA}] /[\mathrm{Mg}]>c a .2) .{ }^{32}$ This result supports the above concept. Such a strong coordination of magnesium with the phosphoramides could easily make the carbon-magnesium bonds of the organomagnesium compounds polar enough to react with St or $\alpha$-MeSt. ${ }^{1,2}$ An increase in the coordinating power of the phosphoramides must result in a further increase in the reactivity of the compounds.

On the other hand, the carbon-metal bonds of organozinc or organoaluminium compounds must be less polar than the bonds of organomagnesium compounds, because the electronegativities of zinc and aluminium atoms are larger than the electronegativity of the magnesium atom: the electronegativities of zinc in diethylzinc and of aluminium in triethylaluminium are reported to be $1.45^{6}$ and $1.59^{6}(1.57)^{5}$, respectively, and Pauling's values for the zinc and aluminium atoms are 1.6 and 1.5 , respectively. ${ }^{37}$ The electronegativity of aluminium in triethylaluminium decreased in the presence of HMPA (1.29 at $[\mathrm{HMPA}] /[\mathrm{Al}]>c a$. 1$),{ }^{5}$ while that of zinc in diethylzinc decreased gradually with increasing [HMPA] $/[\mathrm{Zn}]$ ratio $(1.37,1.34$, and 1.32 at $[\mathrm{HMPA}] /[\mathrm{Zn}]=1.2,2.7$, and 7.3 , respectively). ${ }^{43}$ The extent of decrease in the electronegativities of the aluminium and zinc atoms is smaller than that in the electronegativity of the magnesium atom. This result indicates that the aluminium and zinc atoms in the organometallic compounds behave as less hard acids and do not interact strongly with the phosphoramides. The phosphoramides, therefore, could not make the organoaluminium or organozinc compounds active enough to react with $\mathrm{AN}$. This result also suggests that the interaction of the phosphoramides with the zinc atom is weaker than that with the aluminium atom.

However, if AN is activated, the reaction of the AN with the organometallic compounds must take place. The double bond of AN should be activated by the coordination of the nitrile group of AN with the zinc or aluminium of the organometallic compounds. Actually, it is reported that nitriles interact strongly with Lewis acids such as $\mathrm{AlR}_{3},{ }^{38} \mathrm{AlR}_{n} \mathrm{Cl}_{3-n},{ }^{38}$ and $\mathrm{Zn}\left(\mathrm{C}_{2} \mathrm{H}_{5}\right)_{2} \cdot{ }^{39}$ The polymerization of $\mathrm{AN}$, therefore, is considered to be caused by the coordination of both the phosphoramides and AN to the organometallic compounds. The fact that NIPA was a less efficient donor than HMPA in the polymerization with organoaluminium compounds could be explained by assuming that the bidentate NIPA blocks the coordination site required for the coordination of AN. Such an undesirable coordination of phosphoramides has been already observed in both isomerization of epoxides with $\mathrm{LiBr}-\mathrm{HMPA}$ system ${ }^{40}$ and reactions of epoxides or aldehydes with alkylaluminiums in HMPA. ${ }^{41}$ In the case of the polymerization with diethylzinc, the undesirable coordination of the bidentate phosphoramides may be reduced more than in the polymerization with alkylaluminiums, because of the weak interaction between diethylzinc and the phosphoramides. 
Several complexes of trialkylaluminiums with bidentate donors have been thought to contain 5-coordinated aluminium atoms. ${ }^{42}$ However, it could not be determined whether or not the bidentate phosphoramides used in this study could form such a complex.

The structure of organic moieties of the organometallic compounds is considered to affect the reactivity of the compounds. When the compounds are composed of organic moieties in which there is resonance stabilization, the polarization of the carbon-metal bonds of the compounds must take place more easily. Actually Grignard's reagents derived from benzyl or allyl halides were activated by coordination with HMPA more easily than the reagents derived from alkyl halides. ${ }^{2}$ Moreover, benzylzinc bromide could polymerize not only AN but also methyl methacrylate in the presence of HMPA. ${ }^{43}$ The polymerization with benzyl derivatives such as dibenzylzinc and tribenzylaluminium is now under investigation.

\section{REFERENCES}

1. M. Tomoi and H. Kakiuchi, Kogyo Kagaku Zasshi (J. Chem. Soc. Japan, Ind. Chem. Sect.), 73, 2367 (1970).

2. M. Tomoi and H. Kakiuchi, Polymer J., 5, 195 (1973).

3. M. Tomoi, K. Sekiya, and H. Kakiuchi, ibid., 6, 438 (1974).

4. M. Ikeda, T. Hirano, and T. Tsuruta, Abstracts, SPSJ 23th Annual Meeting, Tokyo, June 5, 1974, p 195 and references therein.

5. A. Akimoto and T. Okada, J. Macromol. Sci.Chem., A7, 1555 (1973).

6. M. Ikeda, T. Hirano, and T. Tsuruta, Makromol. Chem., 150, 127 (1971).

7. J. Furukawa, N. Kawabata, and A. Kato, $J$. Polym. Sci., Part A-1, 5, 3139 (1967).

8. T. Narita, T. Yasumura, and T. Tsuruta, Polymer J., 4, 421 (1973).

9. M.W. G. de Bolster and W. L. Groeneveld, Rec. Trav. Chim., 90, 687 (1971).

10. M. Pianka, J. Appl. Chem., 5, 109 (1955).

11. H. J. Vetter and H. Nöth, Chem. Ber., 96, 1308 (1963).

12. V. Mark, Org. Syntheses, 46, 42 (1966).

13. W. J. Pope and C. S. Gibson, J. Chem. Soc., 101, 735 (1912).

14. H. F. Ebel and B. O. Wagner, Chem. Ber., 104,
307 (1971).

15. R. Noller, "Org. Syntheses Coll. Vol. II," John Wiley \& Sons, Inc., New York, N.Y., 1948, p 184.

16. C. R. Bohn, J. R. Schaejgen, and W. O. Satton, J. Polym. Sci., 55, 531 (1961).

17. H. W. McCormick, ibid., 25, 488 (1957).

18. D. J. Worsfold and S. Bywater, ibid., 26, 299 (1957).

19. J. Leonard and S. L. Malhotra, ibid., Part A-1, 9, 1983 (1971).

20. M.W. G. de Bolster and W. L. Groeneveld, Rec. Trav. Chim., 91, 171 (1972).

21. M.W. G. de Bolster and W. L. Groeneveld, ibid., 91, 95 (1972).

22. M. D. Joesten, M. S. Hussain, and P. G. Lenhert, Inorg. Chem., 9, 151 (1970).

23. M. W. G. de Bolster and W. L. Groeveneveld, Rec. Trav. Chim., 90, 477 (1971) and references therein.

24. M. Levy and M. Szwarc, J. Amer. Chem. Soc., 82, 521 (1960).

25. R. Waack, A. Rembaum, J. D. Coombes, and M. Szwarc, ibid., 79, 2026 (1957).

26. H. L. Hsieh and O. F. McKinney, J. Polym. Sci., Part B, 4, 843 (1966).

27. J. F. Henderson and M. Szwarc, Macromol. Rev., 3, 317 (1968).

28. W. E. Goode, F. H. Owens, R. P. Fellmann, W. H. Synder, and J. E. Moore, J. Polym. Sci., 46, 317 (1960).

29. R. G. Pearson, J. Chem. Educ., 45, 581 (1968); ibid., 45, 643 (1968).

30. W. E. Slinkard and D. W. Meek, Inorg. Chem., 8, 1811 (1969).

31. H. F. Ebel, Tetrahedron, 24, 459 (1968).

32. M. Tomoi and H. Kakiuchi, Bull. Chem. Soc. Japan, 46, 3233 (1973).

33. D. N. Bhattacharyya, C. L. Lee, J. Smid, and M. Szwarc, J. Amer. Chem. Soc., 85, 533 (1963).

34. M. Kurata, H. Okamoto, M. Iwama, M. Abe, and T. Homma, Polymer J., 3, 739 (1972).

35. G. Schwarzenbach, "Die Komplexometrishe Titration," F. Enke Verlag, Stuttgart, 1965.

36. N. L. Zutty and F. J. Welch, J. Polym. Sci., 43, 445 (1960).

37. L. Pauling, "The Nature of the Chemical Bond", Cornell University Press, Ithaca, N.Y., 1960, p 93.

38. T. Mole and E. A. Jeffery, "Organoaluminium Compounds', Elsevier Publishing Co., Amsterdam, 1972, Chapter 12.

39. T. Fujimoto, N. Kawabata, and J. Furukawa, J. Polym. Sci., Part A-1, 6, 1209 (1968). 
Polymerization with Organometallic Compounds

40. B. Rickson, J. Amer. Chem. Soc., 93, 1693 (1971).

41. J. L. Namy, E. Henry-Basch, and P. Freon,
Bull. Soc. Chim. France, 2249 (1970).

42. Ref. 38, Chapter 4.

43. M. Tomoi and H. Kakiuchi, Unpublished results. 